\title{
Design, analysis and testing of an external fixation device manufactured from composite materials
}

\author{
Z. Padovec ${ }^{a, *}$, P. Růžička ${ }^{a}$, R. Sedláček ${ }^{a}$, M. Růžička ${ }^{a}$ \\ ${ }^{a}$ Department of Mechanics, Biomechanics and Mechatronics, Faculty of Mechanical Engineering, Czech Technical University in Prague, \\ Technicka 4, 16607 Prague, Czech Republic
}

Received 5 September 2017; accepted 21 December 2017

\begin{abstract}
An external fixation device (fixator) placed outside the human body is widely used for treating complicated or infected fractures. It is used for several weeks, and its main advantages are that the injury can be treated continuously, and the patient can be fully mobile within a few days. In addition, it allows the doctor to reposition bone fragments during the operation and also during convalescence. Composite materials are used in the structure because of their density/stiffness ratio and also because of their X-ray transparency. Main goal of this work is right design of external fixation device from composites which will be proved by experiments with the focus on fixator's socket. The socket of the fixator is manufactured by press forming from carbon/polyphenylenesulphide (C/PPS) pellets, and the tube for defining the distance between the sockets is manufactured from carbon fiber/epoxy resin (C/epoxy) by filament winding technology. The fixator was designed with the use of finite element calculations, and static and fatigue experiments were successfully performed on two designed configurations.
\end{abstract}

(c) 2017 University of West Bohemia. All rights reserved.

Keywords: external fixator, biomechanics, finite element analysis, static test, fatigue

\section{Introduction}

External fixators are used to fix bones externally during the treatment of fractures or deformities. They are used mainly for long bones and for pelvic bones when treating traumas, and for orthopaedic procedures $[2,8]$. Biocompatible stainless steel bolts are used to anchor the fixator into the bone segments and to maintain them in the desired position. The design of the fixator must be adjustable to the position of the screws and also to appropriate changes in configuration, for example when an adjusting mechanism is used to stimulate the healing process of the bone tissue $[9,15]$.

Widely-used materials for fixators are metals and metal alloys, especially stainless steels, titanium and aluminium alloys (duralumin). At the present time, structures with polymeric materials are used, especially polyetheretherketone (PEEK) or PEEK reinforced with short carbon fibers. With the exception of the screws and wires that are anchored into the bone and that come into contact with soft tissues, an external fixator need not be made from a biocompatible material. However, the material should not be toxic. The fixator must be able to withstand the mechanical loading according to its function, and it must also meet the requirements of clinical practice, e.g. resistance to the steam sterilization process at a temperature of $134^{\circ} \mathrm{C}$ and at a pressure of $304 \mathrm{kPa}$ [19].

A disadvantage of metal materials is their high absorption of X-rays. This complicates radiological examinations of the healing process, as the radiologist has to find a position such

*Corresponding author. Tel.: +420 224352 519, e-mail: Zdenek.Padovec@ fs.cvut.cz. https://doi.org/10.24132/acm.2017.398 
that the fixator does not outshine the investigated bone. A composite material with a polymer matrix is translucent for X-rays, and it does not affect radiographs [22]. This is a useful advantage when dealing with spatially complex fixations, or when investigating the areas around joints.

The mechanical stress in a fixator is defined by the place on human body where the fixator is applied (pelvic bones, lower limb bones, etc.), and by patient activity. An external fixator is maximally loaded during the treatment of a lower-limb long-bone fracture, and the patient is able to walk during the period of convalescence (with the use of locomotion tools, in most cases with crutches) [8]. In the case of a fracture of the tibia, the fixator is loaded by a compressive axial force in the direction of the tibia [15]. In [3], the static load capacity of three types of external fixators was analyzed, and the maximal axial force was determined to be $889 \mathrm{~N}$. In [5], the maximal axial force during cyclic loading of five unilateral fixators and 10000 cycles was evaluated to be $220 \mathrm{~N}$. In [6], the fixators were tested with a static force of $700 \mathrm{~N}$, which was considered to be the average gravitational force of a patient (which is directly proportional to the patient's weight). In [20], a multiplanar (circular) fixator for the tibia was measured in vivo during treatment. The maximal axial force in the fixator was evaluated to be $70 \%$ of the force acting on the ground, but no value was given. In [10], the maximal force acting on the ground was measured as $105 \%$ of the body gravity during walk, i.e. $735 \mathrm{~N}$ for an average patient. The cooperating company PROSPON has own internal standard for external fixation devices and the testing force is set on $1300 \mathrm{~N}$ [17,24]. This loading force is used for fixator verification.

Main goal of this work is modification of existing metal (duralumin) fixator with the use of composite materials and verification of its functionality by static and fatigue tests.

\section{Design description}

Our work focused on the design of the socket of an external fixator for the treatment of long bone fractures to be manufactured from consolidated randomly-oriented C/PPS pellets (see e.g. [4]). The use of this kind of material in this application is quite new. Pellets can be taken from "waste" material, e.g. the material left over after shaping from prepregs and can be cut or ground and reused as a random reinforcement for a new structure [12]. The existing ProSpon UNIFIX external fixator (manufactured from duralumin) was chosen as a model (see Fig. 1). The design of the socket has to take into account the different material properties and the different manufacturing technology when the C/PPS composite with pellets is applied. The supporting tube was manufactured from a composite material with continuous fiber by the filament winding technology (see e.g. [7]). The tube will not be telescopic. The structure is supplemented with sleeves of a spherical joint made from Ti alloy. Between the socket of the fixator and the tube,

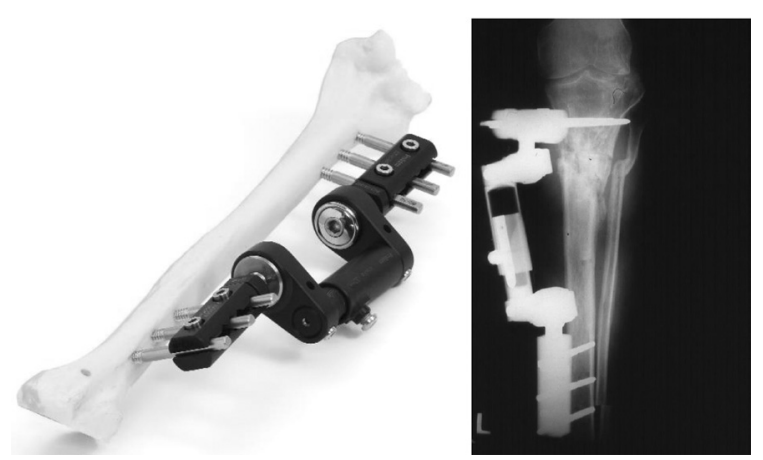

Fig. 1. The ProSpon UNI-FIX unilateral fixator, and a radiograph of a broken tibia with the fixator applied [24] 
and also between socket of the fixator and the sleeve, a bonded joint has been designed with the use of LOCTITE EA 9394 AERO [23].

\section{Finite element analysis}

A computational model of the loaded fixator was made in ABAQUS software. One half of the structure was modelled with symmetric boundary conditions (Fig. 2, left). This model is equivalent to the loading during the experiment which is depicted on scheme in Fig. 2, right. The model consists of the socket of the fixator, the tube and the sleeve for the joint. All parts and adhesive layers were modelled as solid. The connection between the adhesive layer and the other parts was modelled with TIE constraints.

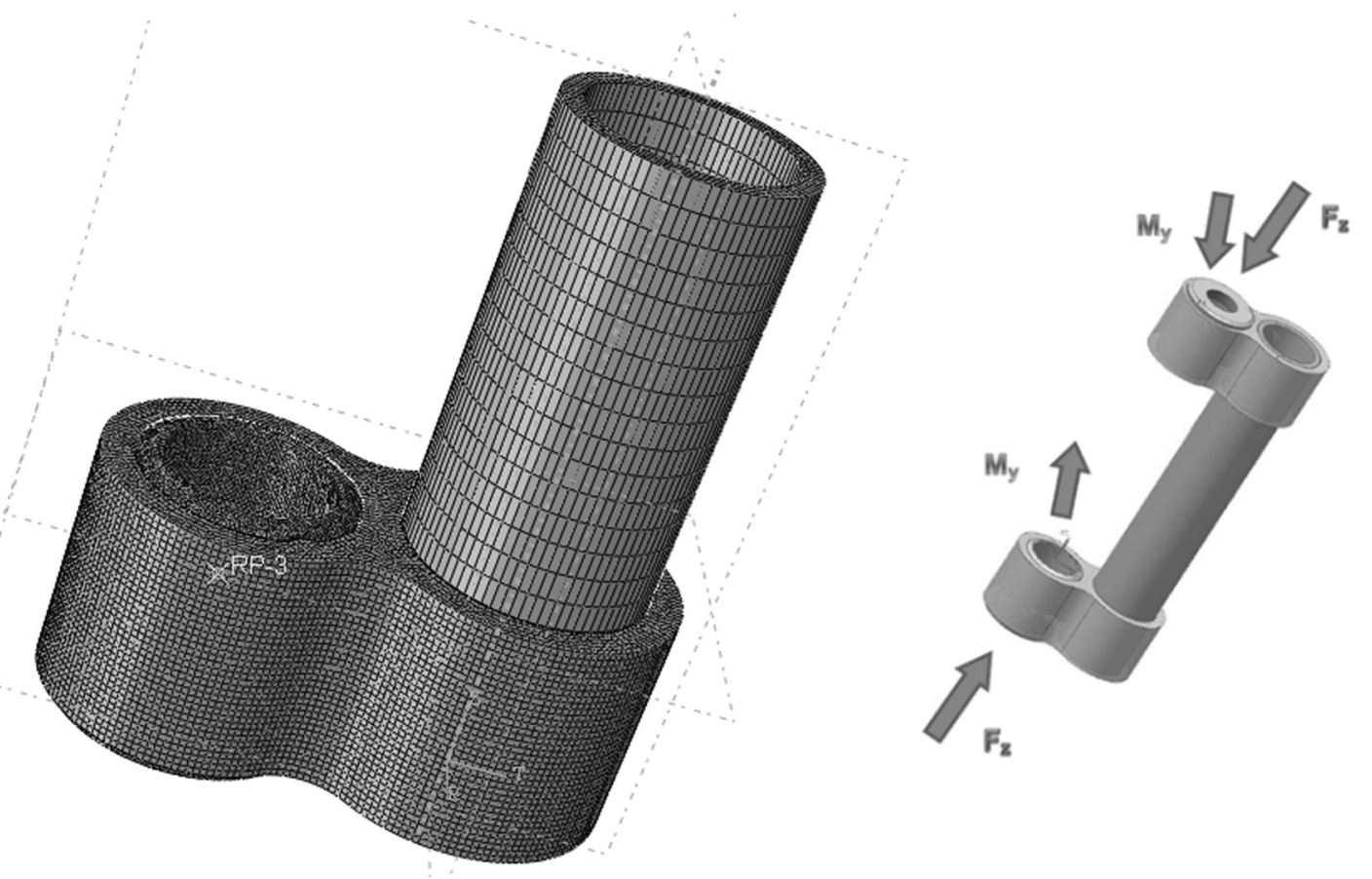

Fig. 2. FE mesh (left) and loading scheme during the experiment (right)

All parts of the model were modelled as linear elastic with the material parameters shown in Tables 1 and 2. Although the socket of the fixator was designed from composite material C/PPS pellets, material model was considered as isotropic because of the random orientation of the pellets (see e.g. [12] for details).

Table 1. Material parameters

\begin{tabular}{|l|r|c|}
\hline & $E[\mathrm{MPa}]$ & $\nu[-]$ \\
\hline C/PPS composite [12] - socket & 34879 & 0.33 \\
\hline Ti alloy - sleeve & 110000 & 0.30 \\
\hline LOCTITE EA 9394 AERO adhesive [23] & 4237 & 0.40 \\
\hline
\end{tabular}

Table 2. Material parameters for a composite tube [14]

\begin{tabular}{|c|c|c|c|c|c|c|c|c|}
\hline $\begin{array}{c}E_{1} \\
{[\mathrm{MPa}]}\end{array}$ & $\begin{array}{c}E_{2} \\
{[\mathrm{MPa}]}\end{array}$ & $\begin{array}{c}E_{3} \\
{[\mathrm{MPa}]}\end{array}$ & $\begin{array}{c}G_{12} \\
{[\mathrm{MPa}]}\end{array}$ & $\begin{array}{c}G_{13} \\
{[\mathrm{MPa}]}\end{array}$ & $\begin{array}{c}G_{23} \\
{[\mathrm{MPa}]}\end{array}$ & $\begin{array}{c}\nu_{12} \\
{[-]}\end{array}$ & $\begin{array}{c}\nu_{13} \\
{[-]}\end{array}$ & $\begin{array}{c}\nu_{23} \\
{[-]}\end{array}$ \\
\hline 46835 & 46835 & 46835 & 13975 & 13975 & 13975 & 0.24 & 0.24 & 0.24 \\
\hline
\end{tabular}


The assumed loading is a compressive axial force $F_{z}$ of $1300 \mathrm{~N}$ in the direction of the tube of the fixator. The bearer of the force is at a distance of $100 \mathrm{~mm}$ from the axis of the socket, and for this reason the substitute force system is used in the computation - axial force $F_{z}$ in the axis of the socket equal to $1300 \mathrm{~N}$, and couples $M_{y}$ equal to $130000 \mathrm{Nmm}$ (Fig. 2, right).

Elements which were used in analysis were linear hexahedral C3D8R and linear tetrahedral C3D4, total number of elements were 247901 , total number of nodes were 178744 . The finite element analysis provides a stress evaluation in a composite socket manufactured by press forming from C/PPS pellets. That is why the simulation is focusing only on behavior of this C/PPS part and not on bone (respectively bone substitution) modelling.

The results of the analysis are presented in Fig. 3, and the maximal von Mises stress value of $76 \mathrm{MPa}$ appears in the lower part of the socket, where tensile stress is dominant. In our case von Mises stress can be used for evaluation because composite socket is considered as isotropic material. Obtained value was compared with the bearing strength from bearing tests on flat C/PPS specimens presented in [13]. Computed value (76 MPa) was 3 to 4 times lower then measured bearing strength. Also during experiments on fixator (described later in the article) there was no observation of bearing failure on this part.

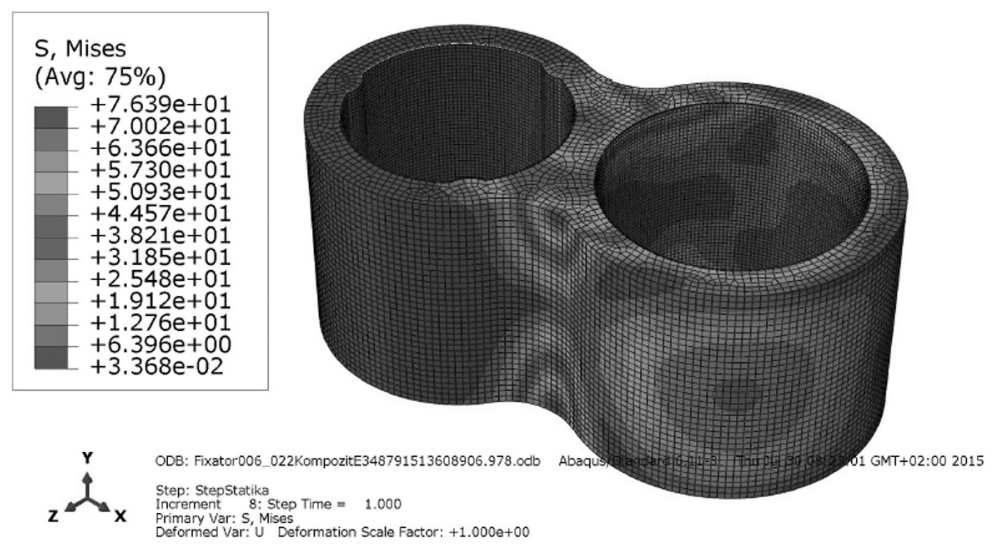

Fig. 3. Results of the FE analysis — von Mises stress

Computation was done for many types of possible design shapes of C/PPS socket and also for varying number of elements with the result, that above-mentioned number of elements is sufficient - when the number of elements was doubled (595871 elements and 554401 nodes) the difference in results (von Mises stress) was $0.47 \%$ [16].

Similar approach to FE modeling was presented in [11] and [21] for example, but the main goal of the works was different. Even the material of fixators analyzed in [11] and [21] was different than in our case (both were manufactured from metal), the methodology of modelling, type of elements, linear elasticity, use of coupling, contact definitions, etc., can be used in our solution.

\section{Test methods}

The tests on the external fixator are performed as quasi-static loading until the axial load reaches $1300 \mathrm{~N}$. The fixator is attached in special jaws (see Fig. 4), and its supporting tube should be at a distance of $100 \mathrm{~mm}$ from the axial loading force. The displacement of the loading part is $40.0 \mathrm{~mm} \cdot \mathrm{min}^{-1}$. After the prescribed load has been reached, there is a $30 \mathrm{~s}$ stay on that load. If the structure has not been destroyed, the assembly is unloaded again at a constant speed of $40.0 \mathrm{~mm} \cdot \mathrm{min}^{-1}$. If the fixator reaches the described load without being destroyed, it is considered to be static verified. 


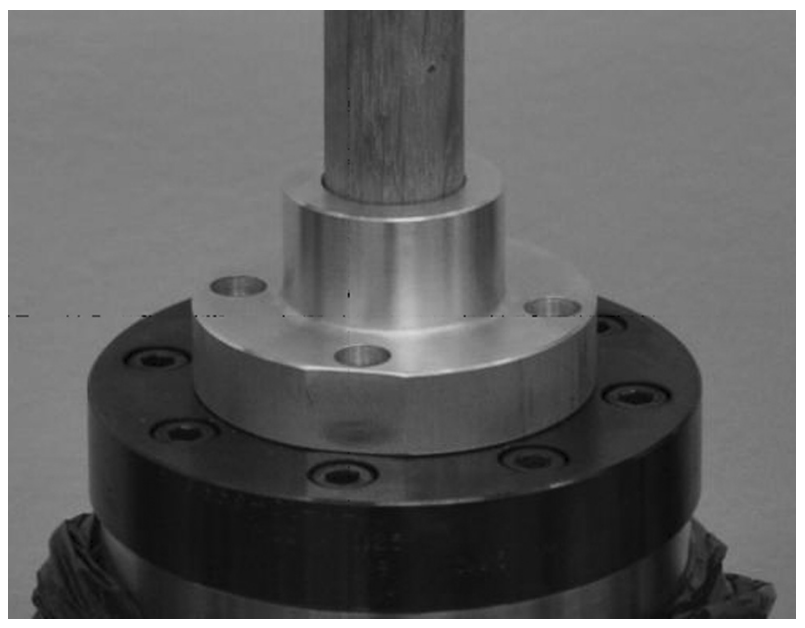

Fig. 4. Special jaws used during the experiment

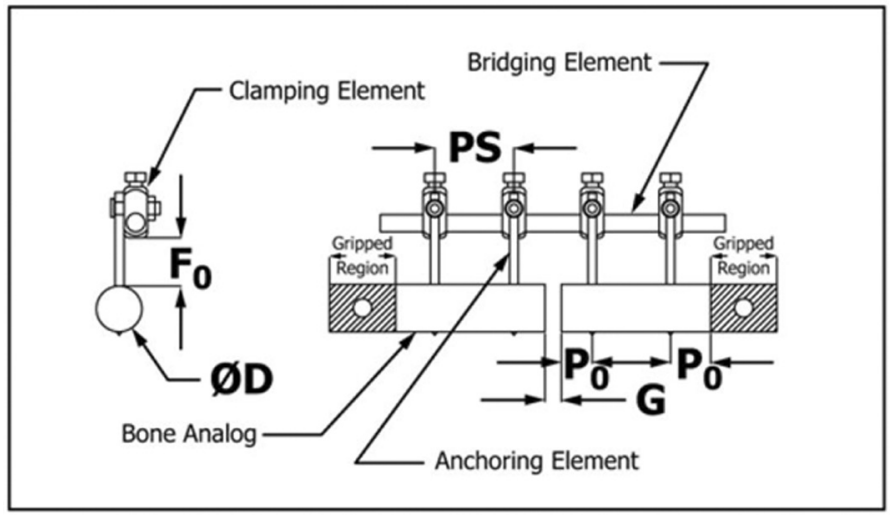

Fig. 5. Loading scheme ASTM F1541-02 [1]

The cyclic testing is based on Gardner method [5] and ASTM standard [1] (see Fig. 5). The fixator should withstand a pulsating force of $220 \mathrm{~N}$ over 10000 cycles. The methodology for cyclic loading developed in our lab required a greater number of cycles -100000 . This requirement is based on the demands placed on the mechanical properties of a femoral or tibial splint, having in mind that the fixator is used primarily in the treatment of long bones. The loading frequency is $5 \mathrm{~Hz}$. Both ends of the bone substitutes are fixed in the jaws (without gripping). The beechwood rods used as bone substitutes are sufficiently tough and this was proofed during the test, when the pins (anchorage elements) were tightly embedded throughout whole test. The parameters monitored during the tests include the displacement amplitude of the loading part. The outcome of the test is a statement that the fixator has withstood the prescribed number of cycles without being destroyed and without malfunctioning.

\section{Experimental}

The tests simulate the loading of an external fixator attached to dislocated bones. Before the experiments begin, the fixator is attached to the bone substitutes, i.e. beechwood rods $35 \mathrm{~mm}$ in diameter [1]. When the fixator is being attached to the wooden rod, it is necessary to set a prescribed distance from the axis of the supporting tube to the loading axis. 

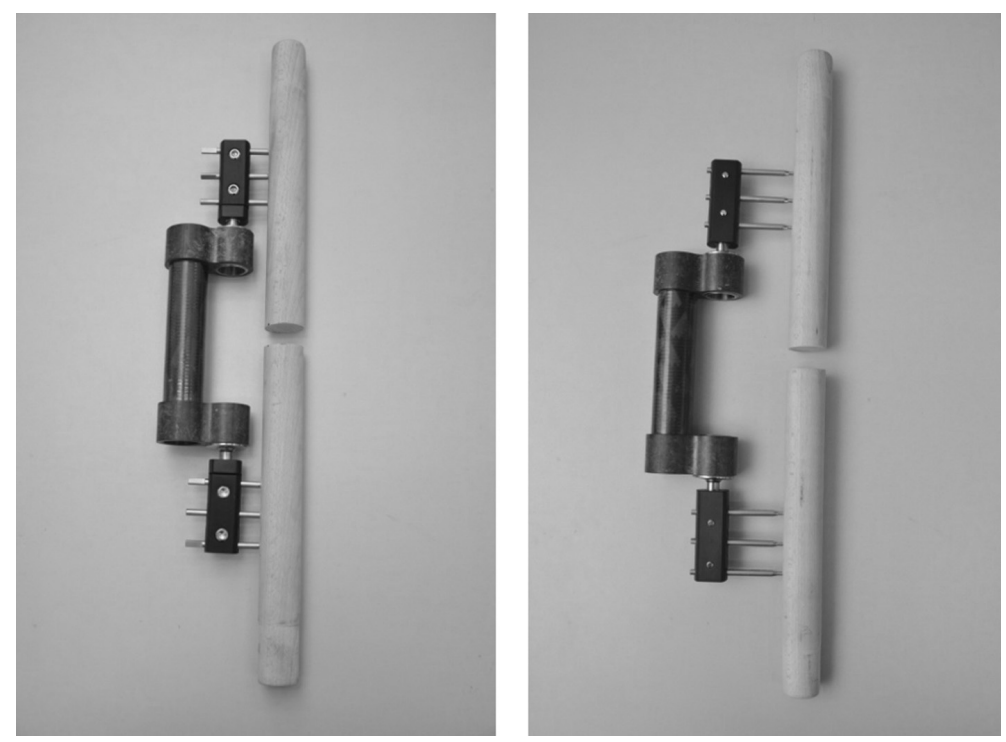

Fig. 6. Tested assembly of external fixator version A (left) and version B (right)

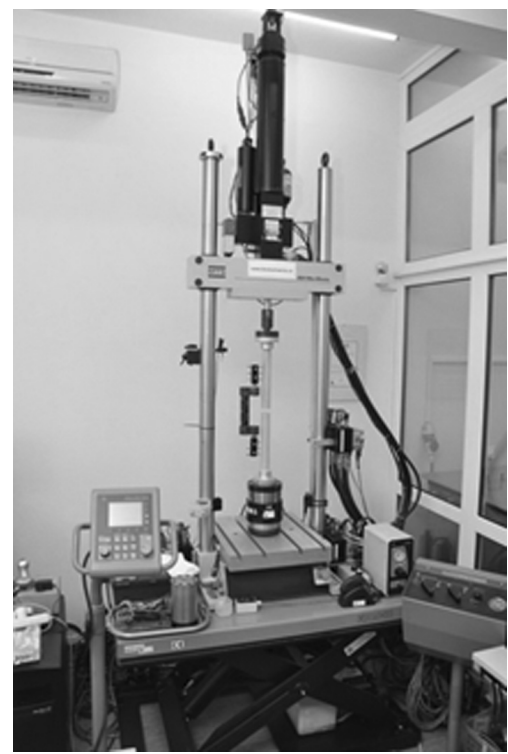

Fig. 7. The MTS 858 Mini Bionix testing system loaded with a specimen

Two versions of the specimens were prepared for the tests (Fig. 6). In version A, conical screws $6 \mathrm{~mm}$ in diameter, $100 / 30 \mathrm{~mm}$ in length and with a $6 / 5 \mathrm{~mm}$ thread (PROSPON PN S0010002) were used for anchoring the fixator to the bone substitutes. The distance between the axis of the supporting tube and the loading axis was $100 \mathrm{~mm}$ (in accordance with the methodology developed at CTU in Prague). In version B, conical screws $6 \mathrm{~mm}$ in diameter, $120 / 40 \mathrm{~mm}$ in length and with a $4.5 / 3.5 \mathrm{~mm}$ thread (PROSPON PN S0010107) were used for anchoring the fixator to the bone substitutes. The distance between the bone substitute and the nearest part of the head of the fixator was $50 \mathrm{~mm}$ (which is equal to $123 \mathrm{~mm}$ distance between the axis of the supporting tube and the loading axis) [1]. Version A is clearly stiffer, because the screws are thicker and there is a shorter distance between the axis of the supporting tube and the loading axis.

The final assembly of the fixator with two wooden rods was clamped into the testing system (Fig. 7) and was loaded (see part 3 for details). Assembly A was loaded, first statically and 
then cyclically. After cyclic loading, static loading was again applied in order to evaluate the maximal axial fixator loading value. The assembly of fixator B was then loaded in the same way. The experiments were performed in the accredited Laboratory of Mechanical Experiments at the Faculty of Mechanical Engineering, CTU in Prague, using the MTS Mini Bionix 858 testing system [25].

\section{Evaluation of the experiments}

The static loading of assembly A proved that the fixator can transfer the prescribed axial load of $1300 \mathrm{~N}$. The relationship between loading force and displacement is shown in Fig. 8, left. The stiffness in compression is comparable with six different hybrid external fixator designs [17] and was mechanically very similar to The Ace, Synthes, Smith \& Nephew Richards, and Howmedica fixators. Cyclic loading of the same assembly shows that assembly A can withstand $10^{5}$ cycles without damage or loss of stiffness. The amplitude of the displacement of the loading part was $0.15 \mathrm{~mm}$, and was unchanged during the course of the test. Then the maximal axial force that the assembly can withstand was evaluated as $3366 \mathrm{~N}$. The similar force was presented by Pugh et al [17] for fixators The Ace, Synthes. During loading, the two parts of the wooden rods came into contact (Fig. 9, left), the spherical joints in the fixator rotated, and the conical screws were permanently deformed (Fig. 9, right). The socket of the fixator remained intact, and it could be re-used, with new conical screws, after the assembly was dismounted.
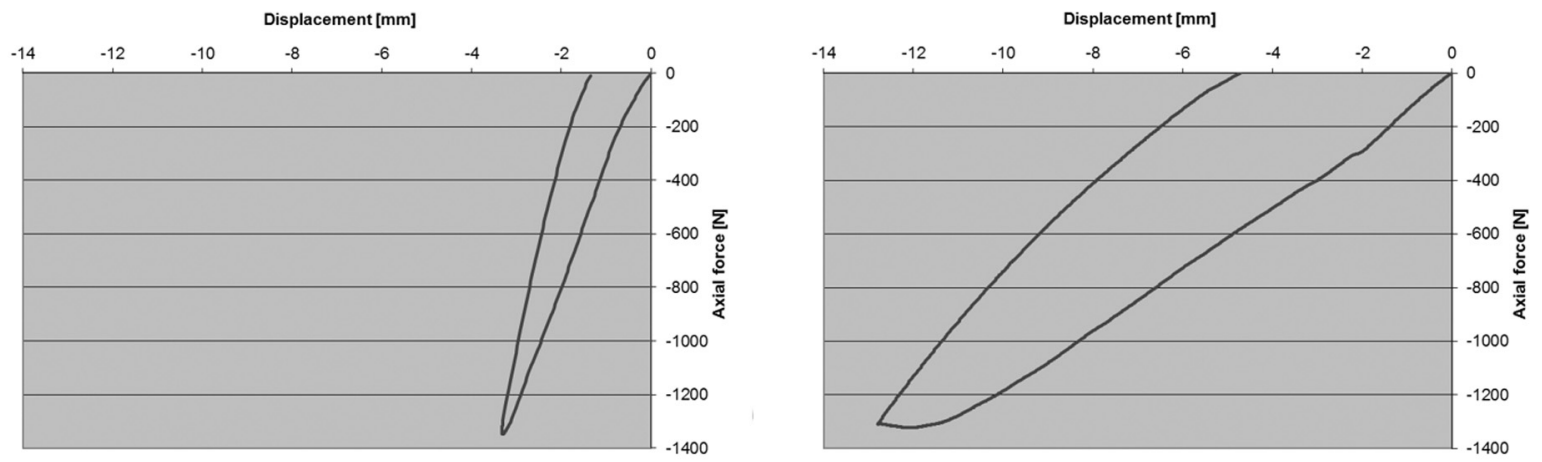

Fig. 8. Relationship between loading force and displacement — version A on the left, version B on the right
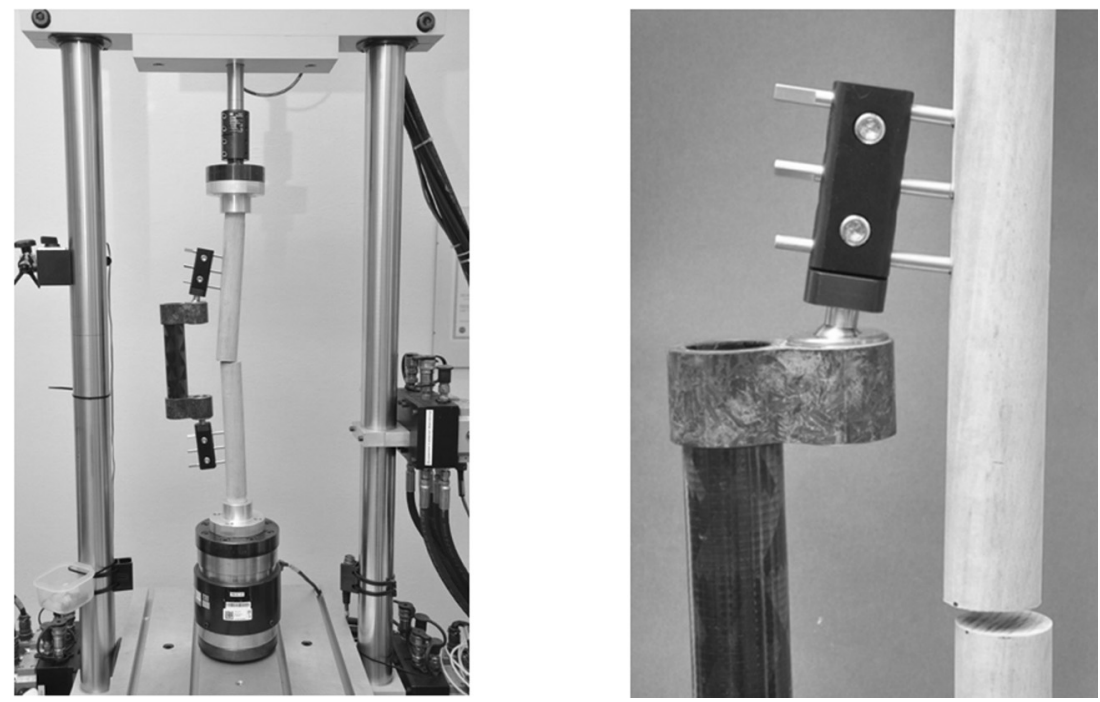

Fig. 9. The wooden rods from assembly A in contact (left); permanent deformation of the conical screws from assembly A (right) 

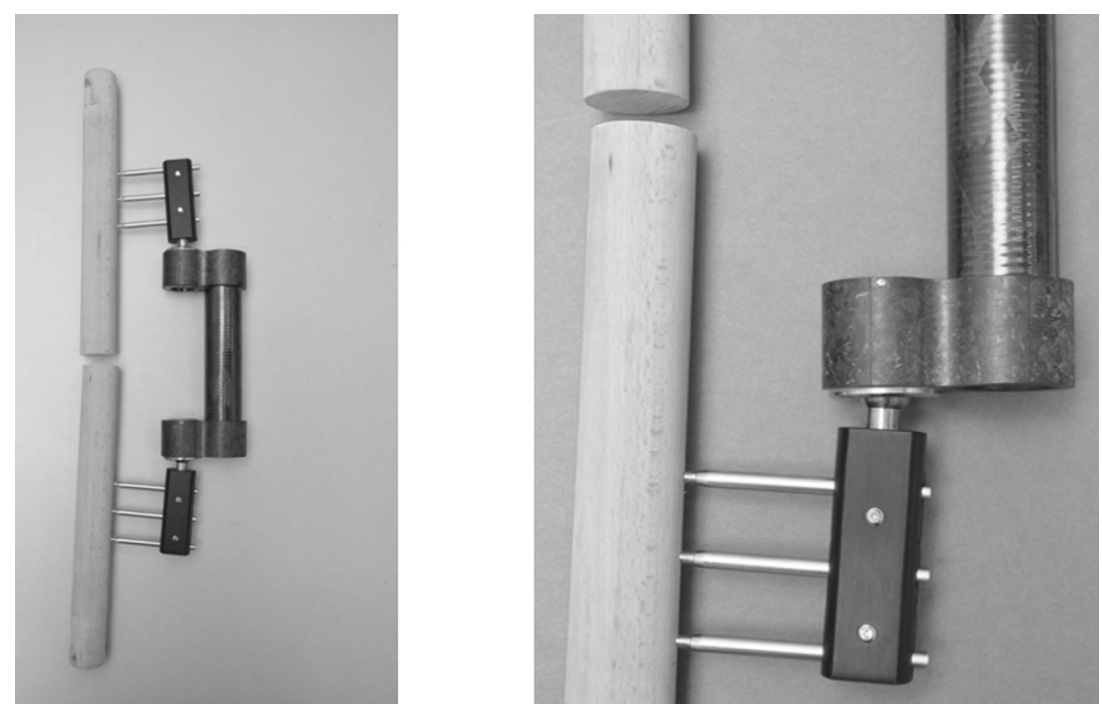

Fig. 10. Complete assembly B after loading (left); permanent deformation of the conical screws from assembly B (right)

During the static loading of assembly B, it was proved that the fixator can also transfer the prescribed axial load of $1300 \mathrm{~N}$. The relationship between the loading force and the displacement is shown in Fig. 8, right. The figure clearly shows that the stiffness of assembly B is lower, but still feasible [17]. This variability of stiffness is acceptable as is presented in [18]. Before cyclic loading, the assembly was dismounted and re-assembled to verify its reusability. Cyclic loading of the same assembly showed that assembly B can withstand $10^{5}$ cycles without damage or loss of stiffness. The amplitude of the displacement of the loading part was $0.68 \mathrm{~mm}$, and did not change during the test. After cyclic loading, the maximal axial force that the assembly could withstand was evaluated as 1365 N. During this loading, the two parts of the wooden rods came into contact, the spherical joints in the fixator rotated slightly (Fig. 10, left), and the conical screws were permanently deformed (Fig. 10, right). The deformation of the conical screws was smaller than in case A. Since there was no damage to the joint locking system or to the new composite parts, it was concluded that the fixator can be re-assembled and re-used.

\section{Conclusions}

Experiments have been carried out to test the strength of a prototype of an external fixator. Two versions of the fixators were assembled for the tests (screws with different threads and of different lengths). The evaluation showed that the external fixator made of composite materials (randomly oriented C/PPS pellets for the socket and wound supporting tube) fulfilled the requirements for the static experiments and also for the cyclic experiments. The carbon composite fixator structure remained intact and functional after the experiments, and was re-usable for further tests.

Finite element analysis was performed to obtain stress values in C/PPS socket which were compared with previous bearing strength experiments. Computed values were lower than measured strength and also no bearing failure was detected during the static and dynamic experiments on fixator.

The concept and the design of the external fracture fixation device made from composite materials has been successfully tested, and it can be further developed for use in clinical practice. 


\section{Acknowledgements}

This work has been supported by project TA03010209 of the Technological Agency of the Czech Republic and by the Grant Agency of the Czech Technical University in Prague, under grant No. SGS15/188/OHK2/3T/12.

\section{References}

[1] ASTM F1541-02 — Standard specification and test methods for external skeletal fixation devices, 2015.

[2] Behrens, F., Searls, K., External fixation of the tibia - basic concepts and prospective evaluation, Journal of Bone and Joint Surgery-British 68 (2) (1986) 246-254.

[3] Caja, V.L., Kim, W., Larsson, S., Chao, E. Y.C., Comparison of the mechanical performance of three types of external fixators: linear, circular and hybrid, Clinical Biomechanics 10 (8) (1995) 401-406. https://doi.org/10.1016/0268-0033(95)00014-3

[4] Feraboli, P., Peitso, E., Deleo, F., Cleveland, T., Stickler, P. B., Characterization of prepreg based discontinuous carbon fiber/epoxy systems, Journal of reinforced plastics and composites 28 (10) (2009) 1191-1214. https://doi.org/10.1177/0731684408088883

[5] Gardner, T. N., Evans, M.,Kenwright, J., A biomechanical study on five unilateral external fracture fixation devices, Clinical Biomechanics 12 (2) (1997) 87-96. https://doi.org/10.1016/S0268-0033(96)00051-4

[6] Gardner, T. N., Simpson, H., Kenwright, J., Rapid application fracture fixators - an evalutaion of mechanical performance, Clinical Biomechanics 16 (2001) 151-159. https://doi.org/10.1016/S0268-0033(00)00071-1

[7] Gay, D., Composite material design and application, Third edition, CRC Press, Boca Raton, 2015.

[8] Giannoudis, P. V., Papakostidis, C., Roberts, C., A review of the management of open fractures of the tibia and femur, Journal of Bone and Joint Surgery-British 88B (3) (2006) 281-289. https://doi.org/10.1302/0301-620X.88B3.16465

[9] Kara, A., Celik, H., Seker, A., Karakoyun, O., Armagan, R., Kuyucu, E., Erdil, M., Treatment of open fractures with a computer-assisted external fixator system without the use of fluoroscopy, Journal of Orthopaedic Surgery and Research 51 (11) (2016). https://doi.org/10.1186/s13018-016-0379-9

[10] Lee, W., Frossard, L., Hagberg, K., Haggstrom, E., Lee Gow, D., Gray, S., Branemark, R., Direct measurement of 3D force and moment on lower-limb osseointegrated fixation, Proceedings of the Symposium on the 3-D Analysis of Human Movement, Valenciennes, France, 2006.

[11] Mešič, E., Avdić, V., Pervan, N., Repčić, N., Finite element analysis and experimental testing of stiffness of the sarafix external fixator, Procedia Engineering 100 (2015) 1598-1607. https://doi.org/10.1016/j.proeng.2015.01.533

[12] Padovec, Z., Růžička, M., Sedláček, R., Král, M., Růžička, P., Comparison of thermoelastic properties of a randomly reinforced composite computed by classical lamination theory and Monte Carlo simulation, Mechanics of Composite Materials 53 (2) (2017) 149-158. https://doi.org/10.1007/s11029-017-9649-9

[13] Padovec, Z., Sedláček, R., Růžička, M., Král, M., Růžička, P., Bearing tests on specimens manufactured from C/PPS pellets, Proceedings of the 54th International Conference on Experimental Stress Analysis EAN 2016, Srní, Czech Republic, 2016.

[14] Product sheet of composite tube from carbon fibre T700 — company COMPO TECH PLUS spol. s r.o., Sušice, CZ.

[15] Pugh, K. J., Wolinsky, P. R., Pienkowski, D., Banit, D., Dawson, J.M., Comparative biomechanics of hybrid external fixation, Journal of Orthopaedic Trauma 6 (13) (1999) 418-425. https://doi.org/10.1097/00005131-199908000-00005 
[16] Růžička, P., et. al., FEM analysis of demonstrator manufactured from pellets, Czech Technical University in Prague [12105/15/12], 2015.

[17] Růzička, P., Sedláček, R., A concept of an external fixator - medical application of composite with thermo-plastic matrix, Czech Technical University in Prague [12105/14/30], 2014.

[18] Schneider, E., Sasse, S., Schmidt, H.G., Schümann, U., Biomechanics of the circular external fixation device - contributions of individual structural elements, Unfallchirurg 11 (95) (1992) 580-587. (in German)

[19] Sedláček, R., Suchý, T., Sucharda, Z., Balík, K., Sochor, M., Šepitka, J., Lukeš, J., The influence of sterilisation process on the micromechanical properties of polyamide fiber-reinforced PDMS composites for orthopaedic applications, Computer Methods in Biomechanics and Biomedical Engineering 15 (2012) 91-92. https://doi.org/10.1080/10255842.2012.713642

[20] Seide, K., Weinrich, N., Wenzl, M.E., Wolter, D., Jürgens, C., Three-dimensional load measurements in an external fixator, Journal of Biomechanics 35 (2004) 1361-1369. https://doi.org/10.1016/j.jbiomech.2003.12.025

[21] Sternick, M. B., Dallacosta, D., Bento, D.Á., Lemos do Reis, M., Relationship between rigidity of external fixator and number of pins: computer analysis using finite elements, Revista Brasileira de Ortopedia 47 (5) (2012) 646-650. https://doi.org/10.1590/S0102-36162012000500017

[22] Suchý, T., Balík, K., Sedláček, R., Sucharda, Z., Sochor, M., Prokop, J., Beneš, J., Křena, J., Radiolucent composites providing high resistance against sterilization decomposition, CeramicsSilikaty 4 (55) (2011) 401-409.

[23] http://www.pccomposites.com/wp-content/uploads/2015/07/PCRS9394-QT_TDS.pdf

[24] http://www.prospon.cz, Internal Standards PN 2015

[25] http://www.upc.edu/sct/documents_equipament/d_77_id-412.pdf 